\title{
ON THE STRUCTURE OF SUPPORT POINT SETS
}

\author{
E. AzOFF \\ Department of Mathematics \\ University of Georgia \\ Athens, Georgia 30602 \\ R. YOUNIS \\ Department of Mathematics \\ Kuwait University \\ P.O. Box 5969 \\ 13060 Safat \\ Kuwait \\ (Received September 7,1988 )
}

ABSTRACT. Let $\mathrm{X}$ be a metrizable compact convex subset of a locally convex space. Using Choquet's Theorem, we determine the structure of the support point set of $X$ when $X$ has countably many extreme points. We also characterize the support points of certain families of analytic functions.

KEY WORDS AND PHRASES: Support point, Extreme point, Choquet's Theorem.

1980 SUBJECT CLASSIFICATION (1985 Revision): Primary: 46A55, 52A07, Secondary: 30 C99.

\section{INTRODUCTION.}

Let $\mathrm{X}$ be a subset of a locally convex space $\mathrm{E}$. A continuous linear functional $\mathrm{J}$ on $\mathrm{X}$ is said to be associated with $f \in X$ if $\operatorname{Re} J(f)=\max \{\operatorname{Re} J(g): g \in X\}$ and $\operatorname{Re} J$ is non constant on $X$. In this case we call $f$ a support point of $X$. The set of support points of $X$ will be denoted by Supp $X$. The set of extreme points of a convex subset $F$ of E will be denoted by Ext $\mathrm{F}$.

Let $D=\{z:|z|<1, z \in C\}$ and equip the space $A$ of functions analytic in $D$ with the topology of uniform convergence on compact subsets of D. This topology is metrizable [1, p.1]. Every continuous linear functional J on A is induced by a sequence $\left\{b_{n}\right\}_{n=0}^{\infty}$ which satisfies $\lim \sup \left|b_{n}\right|^{1 / n}<1$ and $J(f)=\sum_{n=0}^{\infty} a_{n} b_{n}$ for $f(z)=\sum_{n=0}^{\infty} a_{n} z^{n} \in$ A [1, p.36]. Recently, the support points of many subclasses of A have been studied. For more details see [1] and [2].

In Section 2, we consider a metrizable compact convex set $\mathrm{X}$ in a locally convex space. Using Choquet's theorem we determine the structure of Supp $\mathrm{X}$ when Ext $\mathrm{X}$ is countable (Theorem 2.1). 
In Section 3, we consider the classes: $P(p)=\left\{f(z)=\sum_{n=1}^{\infty} a_{n} z^{n} \in A: \sum_{n=1}^{\infty}\left|a_{n}\right|^{p} \leq 1\right\}, 1 \leq p<\infty$. In Theorem 3.4, we determine Supp $P(p)$. Indeed, it is shown that Supp $X$ is in 1-1 correspondence with a proper subset of $\operatorname{Supp} \operatorname{Ball}\left(\ell_{\mathrm{p}}\right)$.

\section{SUPPORT POINTS OF SETS WITH COUNTABLY MANY EXTREME POINTS.}

Let $\mathrm{E}$ be a locally convex space, and suppose that $\mathrm{X}$ is a metrizable compact convex subset of $\mathrm{E}$. A theorem by Choquet $[3, \mathrm{p} .19]$ says that if $\mathrm{x} \in \mathrm{X}$ then there exists a probability measure $\mu_{\mathrm{X}}$ on $\mathrm{X}$, supported by Ext $\mathrm{X}$, such that $L(x)=\int_{E x t ~} L d \mu_{X}$ for every $L$ in $E^{*}$. In case Ext $X$ is countable (possibly finite), we have the following:

CIIOQUET'S THEOREM (Countable Case). Suppose Ext $X=\left\{f_{n}\right\}$ is countable. Then $X=\left\{\sum_{n} \lambda_{n} f_{n}: \lambda_{n} \geq 0\right.$ for each $\mathrm{n}$ and $\left.\sum_{\mathbf{n}} \lambda_{\mathbf{n}}=1\right\}$.

PROOF. Let $f \in X$. By Choquet's Theorem, there exists a probability measure $\mu_{f}$ on $X$, supported by $\left\{f_{n}\right\}$, such that $L(f)=\int_{\left\{f_{n}\right\}} L d \mu_{f}$. Thus $L(f)=\sum_{n} \mu_{f}\left(f_{n}\right) L\left(f_{n}\right)$. Hence $L\left(f-\sum_{n} \mu_{f}\left(f_{n}\right) f_{n}\right)=0$.

Since this is true for every $L$ in $E^{*}$, we get $f=\sum_{n} \mu_{f}\left(f_{n}\right) f_{n}$, as required.

We proceed to the main result of this section.

THEOREM 2.1. Let $\mathrm{X}$ be a metrizable compact convex subset of a locally compact space $\mathrm{E}$ such that Ext $X=\left\{f_{n}\right\}$ is countable. For each positive integer $n$, set $K_{n}$ equal to the closed convex hull of $\left\{f_{i}: i \neq n\right\}$. Then

(1) Supp $X$ is contained in the union of those $K_{n}$ which are proper subsets of $X$.

(2) $K_{n} \subseteq$ Supp $X$ if and only if $f_{n} \notin$ closed affine hull of $\left\{f_{i}: i \neq n\right\}$.

PROOF. To prove (1), let $f \in \operatorname{Supp} X$. By Choquet's Theorem, we can write $f=\sum_{i} \lambda_{i} f_{i}$ with each $\lambda_{i} \geq 0$ and $\sum_{i} \lambda_{i}=1$. Let $\phi$ be a continuous linear functional associated with $f$. Then $\operatorname{Re} \phi(f)=\sum \lambda_{i} \operatorname{Re} \phi\left(f_{i}\right)$ $\leq \sum_{i} \lambda_{i} \operatorname{Re} \phi(f)=\operatorname{Re} \phi(f)$. Hence we must have $\operatorname{Re} \phi\left(f_{i}\right)=\operatorname{Re} \phi(f)$ whenever $\lambda_{i}>0$. On the other hand, since $\operatorname{Re} \phi$ is non-constant on $X$, we must have $\operatorname{Re} \phi\left(f_{i}\right) \neq \operatorname{Re} \phi(f)$ for some $i$. We conclude that $\lambda_{i}=0$ for some $i$, as required.

To prove (2), suppose that $f_{n}$ does not belong to the closed affine hull $H$ of $\left\{f_{i}: i \neq n\right\}$ and fix $g \in K_{n}$. Then $H-g$ is a closed real subspace of $E$ not continuing $f_{n}-g$. A version of the Hahn-Banach theorem [4, page 59] gives a functional $J$ in $E^{*}$ whose real part $\phi$ vanishes on $H-g$ while $\phi\left(f_{n}-g\right)=-1$. Set $\phi\left(f_{n+1}\right)=b$. Then $\phi\left(f_{n}\right)=b-1$ while $\phi\left(f_{i}\right)=\phi\left(f_{n+1}\right)=b$ for every $i \neq n$. Thus, $\phi(g)=b$ for all $g \in K_{n}$. For any $h$ in $X$. by Choquet's Theorem, we have $\mathrm{h}=\sum_{\mathrm{i}} \beta_{\mathrm{i}} \mathrm{f}_{\mathrm{i}}$ with $\beta_{\mathrm{i}} \geq 0$ and $\sum_{\mathrm{i}} \beta_{\mathrm{i}}=1$. Thus $\phi(\mathrm{h})=\beta_{\mathrm{n}}(\mathrm{b}-1)+\sum_{\mathrm{i} \neq \mathrm{n}} \beta_{\mathrm{i}} \mathrm{b}$ $=\mathrm{b}-\beta_{\mathrm{n}} \leq \mathrm{b}$. This shows that $\mathrm{g} \in \operatorname{Supp} \mathrm{X}$.

Conversely, assume that $K_{n} \subseteq$ Supp $X$. For ease of notation we take $n=1$ and assume Ext $X=\left\{f_{n}\right\}_{n=1}^{\infty}$ is infinite. By assumption, $f=\sum_{i=2}^{\infty} \frac{1}{2^{i-1}} f_{i}$ is a support point of $X$. Let $\phi$ be an associated linear functional in $E^{*}$ and set $S=\{g \in E: \operatorname{Re} \phi(g)=\operatorname{Re} \phi(f)\}$. Note that $S$ is a closed affine subspace of $E$. Since $\operatorname{Re} \phi(f)=$ $\sum_{i=2}^{\infty} \frac{1}{2^{i-1}} \operatorname{Re} \phi\left(f_{i}\right) \leq \sum_{i=2}^{\infty} \frac{1}{2^{i-1}} \operatorname{Re} \phi(f)=\operatorname{Re} \phi(f)$, we have $\operatorname{Re} \phi\left(f_{i}\right)=\operatorname{Re} \phi(f)$ for all $i \geq 2$. Thus the closed affine hull 
of $\left\{f_{i}: i \neq 1\right\} \subseteq S$. On the other hand, in view of Choquet's Theorem, if $f_{1} \in S$ then Re $\phi$ would be constant on $X$. Thus $f_{1} \notin S$ and consequently, $f_{1} \notin$ closed affine hull of $\left\{f_{i}: i \neq 1\right\}$.

EXAMPLES. (1) Let $\mathrm{X}$ be a triangle in $\mathrm{R}^{2}$ with vertices $\mathrm{f}_{1}, \mathrm{f}_{2}$ and $\mathrm{f}_{3}$. These vertices are the extreme points of $\mathrm{X}$ and the affine hull of any two of them is a line, not containing the third. The theorem guaranters that Supp $X=\bigcup_{n=1}^{3} K_{n}$, which is indeed the boundary of $X$.

(2) Let $X$ be a square in $R^{2}$ with vertices $f_{1}, f_{2}, f_{3}$ and $f_{4}$. The affine hull of any three of the $f_{i}$ 's is all of $R^{2}$. In particular, each $f_{i} \in$ affine hull of $\left\{f_{j}: j \neq i\right\}$. The theorem guarantees that no $K_{n}$ is contained in Supp $X$. In fact, Supp $\mathrm{X}=$ the boundary of $\mathrm{X}$ has no interior.

(3) Let $T$ be the family of all functions which are analytic and univalent in $D$, and take the form $f(z)=$ $z-\sum_{n=2}^{\infty} a_{n} z^{n}, a_{n} \geq 0$. By [5], Ext. $T=\left\{f_{n}\right\}_{n=1}^{\infty}$, where $f_{1}(z)=z$ and $f_{n}(z)=z-\frac{1}{n} z^{n}$ for $n>1$. For $n>1$, it is clear that $f_{n}$ does not belong to the closed affine hull of the remaining $\left\{f_{i}\right\}$, so $\bigcup_{n=2}^{\infty} K_{n} \subseteq$ Supp $X$ by the second part of the Theorem. Since $f_{1}$ is a limit point of the remaining $f_{i}^{\prime}, K_{1}=X$ and $\stackrel{n=2}{S u p p} X=\bigcup_{n=2}^{\infty} K_{n}$ by the first part of the Theorem.

COROLlary 2.2. Let $\mathrm{X}$ be as in Theorem 2.1. Then Supp $\mathrm{X}=\bigcup_{\alpha} \overline{c o}\left(\mathrm{E}_{\alpha}\right)$, where each $\mathrm{E}_{\alpha}$ is a subset of Ext $X$.

PROOF. Suppose $f \in \operatorname{Supp} X$ and $\phi$ is an associated linear functional with $f$. Writing $f=\sum_{i} \lambda_{i} f_{i}$, we sec that $\operatorname{Re} \phi(f)=\operatorname{Re} \phi\left(f_{i}\right)$ whenever $\lambda_{i} \neq 0$. Take $E_{\alpha}=\left\{f_{i} \mid \lambda_{i} \neq 0\right\}$. Then $f \in \overline{c o}\left(E_{\alpha}\right) \subseteq \operatorname{Supp} X$.

The theorem says these $E_{\alpha}$ are proper subsets of Ext X, i.e., they cannot be "too big". The next proposition implies that they can't all be singletons, i.e., "too small” .

PROPOSITION 2.3. Let $\mathrm{X}$ be a compact convex subset of a locally convex space. If $\mathrm{X}$ has more than two extreme points, then Supp $\mathrm{X}$ is uncountable.

PROOF. Without loss of generality we may assume that $0 \in X$. Let $f_{1}$ and $f_{2}$ be two independent cleinents of $\mathrm{X}$, and let $\phi_{1}$ and $\phi_{2}$ be continuous and linear functionals such that $\phi_{1}\left(\mathrm{f}_{1}\right)=\phi_{2}\left(\mathrm{f}_{2}\right)=1$ and $\phi_{1}\left(\mathrm{f}_{2}\right)=\phi_{2}\left(\mathrm{f}_{1}\right)=0$. Define $\psi: \mathrm{X} \rightarrow \mathrm{R}^{2}$ by $\psi(\mathrm{f})=\left(\phi_{1}(\mathrm{f}), \phi_{2}(\mathrm{f})\right)$. Then $\psi(\mathrm{X})$ is a compact convex subset of $\mathrm{R}^{2}$ with non empty interior. Since $\psi(X)$ has uncountably many boundary points, $\operatorname{Supp}(\psi(X))$ is uncountable. Since $\psi^{-1}$ takes support points to support points, we see that Supp $\mathrm{X}$ is uncountable too.

EXAMPLE. Take $f_{n}=e^{\frac{2 \pi i}{n}}$ for $n=1,2, \ldots$ and $X=\overline{c o}\left\{f_{n}\right\}$ in $R^{2}$. Then Supp $X=\bigcup_{n=1}^{\infty}$ co $\left\{f_{n}, f_{n+1}\right\}$. IIere all the $\mathrm{E}_{\alpha}$ 's have cardinality two even though Ext $\mathrm{X}$ is infinite.

COROLLARY 2.4. Let $\mathrm{X}$ be as in Theorem 2.1. Then Ext $\mathrm{X}=\operatorname{Supp} \mathrm{X}$ if and only if $\mathrm{X}$ has two cxtreme points. 


\section{SUPPORT POINTS OF CERTAIN CLASSES OF ANALY'TIC FUNCTIONS.}

For $1 \leq p<\infty$, define $P(p)=\left\{\sum_{n=1}^{\infty} a_{n} z^{n} \in A: \sum_{n=1}^{\infty}\left|a_{n}\right|^{p} \leq 1\right\}$. It is easy to see that the classes $P(p)$ are compact convex subsets of $A$. These classes are closely related to Ball $\left(\ell_{p}\right)$ and we will find that $S u p p P(p)$ is in oneto-one correspondence with a proper subset of $\operatorname{Supp} \operatorname{Ball}\left(\ell_{\mathrm{p}}\right)$. As a corollary, we determine the support points of certain families of univalent functions. We use the notation a for the sequence $\left\{a_{n}\right\}_{n=1}^{\infty}$.

We begin with a simple observation.

PROPOSITION 3.1. Let $X$ be the unit ball of a Banach space $E$. Then Supp $X=\{x \in X:\|x\|=1\}$. If $\phi$ is associated with $\mathrm{x}$, then $\phi(\mathrm{x})=\|\phi\|$.

PROOF. That every vector of norm one belongs to Supp $X$ is a consequence of the Hahn-Banach theorem. Suppose conversely that the real part of $\phi \in X^{*}$ achieves its maximum over $X$ at $x$. Since $X$ is closed under multiplication by scalars of absolute value at most one, we have $\operatorname{Re} \phi(x)=\sup _{y \in X} \operatorname{Re} \phi(y)=\|\phi\|$. Thus $\|\phi\|=\operatorname{Re} \phi(x)$ $\leq\|\phi\|\|x\|$ and so $\|x\|=1$. Moreover $\operatorname{Re} \phi(x)=\|\phi\|$ implies $\operatorname{Re} \phi(x) \geq|\phi(x)|$, so $\phi(x)$ is in fact real.

EXAMPLE. The family $P(p)$ “looks like" the unit ball of $\ell_{p}$, but we cannot immediately apply Proposition 3.1 to find its support points. For example, the sequence $\left\{a_{n}\right\}_{n=1}^{\infty}=\left\{\sqrt{\frac{6}{\pi}} \frac{1}{n}\right\}_{n=1}^{\infty}$ belongs to the unit sphere of $\ell_{2}$, but $\sum_{n=1}^{\infty} a_{n} z^{n}$ is not a support point of $P(2)$. The problem is that any non-constant linear functional $\left\{b_{n}\right\}_{n=1}^{\infty} \in e_{2}^{*}$ which assumes its maximum at $\left\{a_{n}\right\}_{n=1}^{\infty}$ must be a scalar multiple of $\left\{a_{n}\right\}_{n=1}^{\infty}$. So lim $\sup \sqrt[n]{\left|b_{n}\right|}=1$, which does not correspond to a continuous linear functional on $\mathrm{A}$.

We find the support points of $P(p)$ by making the remarks in the preceeding example more precise.

PROPOSITION 3.2. Suppose $\mathrm{T}: \mathrm{E} \rightarrow \mathrm{F}$ is a linear, injective, and continuous map between topological vector spaces $E$ and $F$, and let $X$ be a subset of $E$. Then $T x \in$ Supp TX if and only if $x \in$ Supp $X$ and some linear functional associated with $\mathrm{x}$ belongs to range $\mathrm{T}^{*}$.

PROOF. Recall that $\mathrm{T}^{*}: \mathrm{F}^{*} \rightarrow \mathrm{E}^{*}$ is defined by $\mathrm{T}^{*} \psi=\psi \circ \mathrm{T}$. Suppose $\mathrm{Tx} \in \mathrm{Supp} \mathrm{TX}$ and choose $\psi \in \mathrm{F}^{*}$

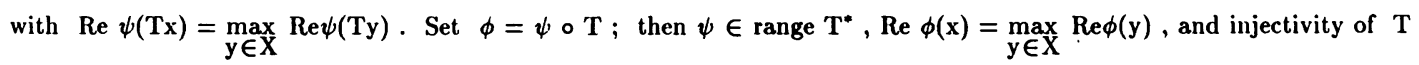
implies that $\operatorname{Re} \phi$ is not constant on $X$.

Conversely, let $\phi \in$ range $\mathrm{T}^{*}$ such that $\operatorname{Re} \phi(\mathrm{x})=\max _{\mathrm{y} \in \mathrm{X}} \operatorname{Re} \phi(\mathrm{y})$. Write $\phi=\psi \circ \mathrm{T}, \psi \in \mathrm{F}^{*}$. Then $\operatorname{Re} \psi(\mathrm{Tx})=\max _{\mathrm{y} \in \mathrm{TX}} \psi(\mathrm{y})$, and $\operatorname{Re} \psi$ cannot be constant on $\mathrm{TX}$ since $\operatorname{Re} \phi$ is not constant on $\mathrm{X}$.

PROPOSITION 3.3. Let $a \in X=\operatorname{Ball}\left(\ell_{p}\right),(1<p<\infty)$, with $\|\mathrm{a}\|_{\mathrm{p}}=1$, and $\mathrm{b} \in \ell_{\mathrm{q}}$. Then:

(1) If $b$ is associated with $a$, then there exists $\beta \neq 0$ with $\beta\left|b_{n}\right|^{q}=\left|a_{n}\right|^{p}$ for all $n$.

(2) If $b_{n}= \begin{cases}\frac{a_{n}}{\left|a_{n}\right|}\left|a_{n}\right|^{p-1} & \text { if } a_{n} \neq 0 \\ 0 & \text { otherwise }\end{cases}$ then $b$ is associated with $a$. 
PROOF. (1) From Proposition 3.1, we learn that $b(a)=\|b\|_{q}=\|b\|_{q}\left\|_{a}\right\|_{p}$. Thus we have Holder cquality, so there exists $\beta \neq 0$ with $\beta\left|b_{n}\right|^{1}=\left|a_{n}\right|^{\prime \prime}$ for all $n$.

() $\quad b(a)=\sum a_{n} b_{n}=\sum\left|a_{n}\right|\left|a_{n}\right|^{p-1}=\sum\left|a_{n}\right|^{p}=1$, while $\|b\|_{q}=\sum_{n=1}^{\infty}\left|a_{n 1}\right|^{(p-1) a_{1}}=1$, so this result follows from IIolder's inequality.

The following is the main result of this section.

THEOREM 3.4. L.e $f(z)=\sum_{n=1}^{x} a_{n} z^{n}$ be in $P(p)$. Then $f$ is a support point of $P(p)$ if and only if

(1) $r$ is analytic in $\bar{D}$ and $\sum_{n=1}^{n=1}\left|a_{n}\right|^{p}=1$, for $1<p<\infty$.

(2) $f(x)=\sum_{n=1}^{N} a_{n} z^{n}$, where $N$ is some positive integer and $\sum_{n=1}^{N}\left|a_{n}\right|=1$ for $p=1$.

ProOf. Define $\mathrm{I}: \ell_{\mathrm{P}} \rightarrow \Lambda$ by $\mathrm{T}(\mathrm{a})=\sum_{\mathrm{n}=1}^{\infty} \mathrm{a}_{\mathrm{n}} z^{\mathrm{n}}$. Clearly $\mathrm{T}$ maps Ball $\left(\ell_{\mathrm{p}}\right)$ onto $\mathrm{P}(\mathrm{p})$ and $\mathrm{T}$ is injertive. Moreover for any $r<1$ and $a \in C_{p},(1<p<\infty)$, we have $\sup _{|z| \leq r}|'(a)(z)| \leq \sum_{n=1}^{\infty}\left|a_{n}\right| r^{n} \leq\|a\|_{1},\left(\frac{1}{1-r^{9}}\right)^{1 / 9}$, by Hôlder's incquality, so $\mathrm{T}$ is continuous. Similarly for $\mathrm{p}=1$.

If $\phi \in A^{*}$ is given by $\phi\left(\sum_{n=1}^{\infty} a_{n} z^{n}\right)=\sum_{n=1}^{\infty} a_{n} b_{n}$. then $\left(T^{*} \phi\right)(a)=\phi(T a)=\sum_{n=1}^{\infty} a_{n} b_{n}$ for every a $\in c_{1}$. So $T^{*} \phi$ is the sequence $\left\{b_{n}\right\}_{n=1}^{\infty}$ considered as a member of $\left(\ell_{p}\right)^{*}=\ell_{q}$. Thus $\left\{b_{n}\right\}_{n=1}^{\infty} \in\left(\ell_{p}\right)^{*}$ is in the range of $1^{*}$ if and only if $\lim \sup \sqrt[n]{\left|b_{n}\right|}<1$.

(1) Suppose $\Gamma=$ Ta $\in \operatorname{Supp} P(\mathbf{p})$. By Proposition 3.2, a $\in$ Supp Ball $\left(\ell_{\mathrm{p}}\right)$. 'Thus by Proposition 3.1, we get. $\sum_{n=1}^{\infty}\left|a_{n}\right|^{p}=1$. If the functional associated with $T a$ is given by $\left\{b_{n}\right\}_{n=1}^{\infty}$, then $\lim \sup n \sqrt{\left|b_{n}\right|}<1$. By Proposition 3.3 , there exists $\beta \neq 0$ such that $\left|a_{n}\right|^{p}=\beta\left|b_{n}\right|^{q}$ for all $n$. Thus lim sup $n \sqrt{\mid a_{n} n} \mid<1$ and so $f$ is analytic in $\bar{D}$.

Conversely, suppose that $f==T(a)$ is analytic in $\bar{D}$ with $\sum_{n=1}^{\infty}\left|a_{n}\right|^{p}=1$. Then a $\in \operatorname{Supp}$ Ball $\left(\ell_{p}\right) \quad b y$ Proposition 3.1, and one can choose the functional associated with a as in the formula of Proposition 3.3. Since the radius of convergence of the power series of $f$ is greater than one, $\lim \sup n \sqrt{\left|a_{n}\right|}<1$ so $\lim \sup n \sqrt{\left|b_{n}\right|}<1$ and thus $b \in$ range $\mathrm{I}^{*}$. Thus $f \in \operatorname{Supp} \mathrm{I}^{\prime}(\mathrm{p})$ by Proposition 3.2 .

(2) Suppose $\mathrm{f}=\mathrm{Ta} \in \operatorname{Supp} \mathrm{P}(1)$ and $\mathrm{b}$ is a functional associated with a. Then $\|\mathrm{a}\|_{1}=1$ and $\mathrm{b}(\mathrm{a})=\|\mathrm{b}\|_{\infty}$ by Propositions 3.2 and 3.1. Thus equality must hold at all points of the chain $b(a) \leq \sum_{n=1}^{\infty}\left|a_{n}\right|\left|b_{n}\right| \leq \sum_{n=1}^{\infty}\left|a_{n}\right| \|\left. b\right|_{\infty}$ $\leq\|b\|_{\infty}$. In particular $\left|b_{n}\right|=\|b\|_{\infty}$ whenever $a_{n} \neq 0$. Since $\lim \sup n \sqrt{\left|b_{n}\right|}<1$, this ueans $a_{n}=0$ for all but finitely many $\mathbf{n}$, as required

Conversely, suppose $\mathrm{Ta}=\mathrm{f}(\mathrm{z})=\sum_{n=1}^{N} a_{n} z_{n}$ and $\sum_{n=1}^{N}\left|a_{n}\right|=1$. Then $a \in \operatorname{Supp}$ Ball $\left(\ell_{1}\right)$.

Define $b_{n} \equiv \begin{cases}\frac{a_{n}}{\left|a_{n}\right|} & \text { if } a_{n} \neq 0 \\ 0 & \text { otherwise }\end{cases}$

Then $\lim \sup n \sqrt{\left|b_{n}\right|}<1$ and $\left\{b_{n}\right\}_{n=1}^{\infty} \in\left(\ell_{p}\right)^{\prime}$ is associated with a By Propesition 2.2, i is a suppon! point of $P(1)$, as required.

Let $Q(p)=\left\{\mathrm{f}(\mathrm{z})=\mathrm{z}+\sum_{n=2}^{\infty} a_{11} z^{n} \in \mathrm{A}:\left.\sum_{1:-2}|n| \mathrm{a}_{\mathrm{n}}\right|^{p} \leq 1\right\}, 1 \leq \mathrm{p}<\infty$. The class $Q(1)$ has bren sludied in [6]. We remark that each element of $Q(1)$ is mivalent. 
COROLLARY 3.5. A function $f(z)=z+\sum_{n=2}^{\infty} a_{11} z^{n}$ is a support point of $Q(p)$ if and only if

(1) $f$ is analytic in $\bar{D}$ and $\sum_{n=2}^{\infty} n\left|a_{n}\right|^{p}=1$, if $1<p<\infty$

(2) $f(z)=z+\sum_{n=2}^{N} a_{n} z^{n}$ and $\sum_{n=2}^{N}\left|a_{n 1}\right|=1$, for some positive integer $N \geq 2$, if $p=1$.

PROOF. One way to see this, is to replace $\ell_{p}$ by $\ell_{p}(\mu)$, where $\mu(n)=n, n=2.3 \ldots$. in the proof of Theorem 3.4.

REMARK. One can define $P(\infty)=\left\{f(z)=\sum_{n=1}^{\infty} a_{n} z^{n}:\right.$ sup $\left.\left|a_{n}\right| \leq 1\right\}$. One can show, using an argument similar to the proof of Theorein 3.4, that Supp $P(\infty)=\left\{f(z)=\sum_{n=1}^{\infty} a_{n} z^{n}:\left|a_{n}\right|=1\right.$ for some $\left.n \geq 1\right\}$.

\section{REFERENCES}

1. SCIIOBER, G. Univalent Functions - Selected Topics, Springer-Verlag, 1975.

2. IlALLenBECK, D.J. and MACGREGOR, T.H. Linear Problems and Convexity Techuiques in Geometric Function 'Theory, Pitman, 1984.

3. PHELPS, R.R. Lectures on Choquet's 'T'heorem, Van Nostrand, 1966.

4. RUDin, W. Functional Analysis, McGraw-Hill, 1973.

5. SILVERMaN, H. Univalent functions with negative coefficients, Proc. Amer. Math. Soc. 51(1975), 109-116.

6. SILVERMAN, H. Order starlikeness for multipliers of univalent functions, J. Math. Anal. Appl. 103(1984) 48-57. 


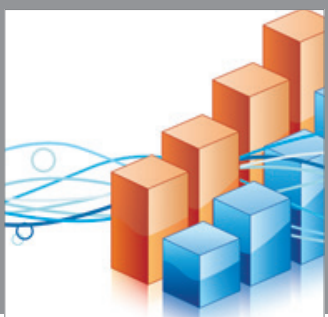

Advances in

Operations Research

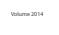

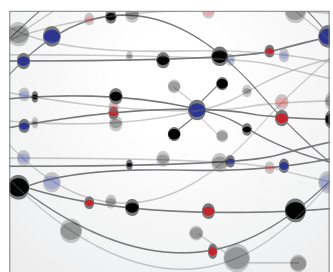

\section{The Scientific} World Journal
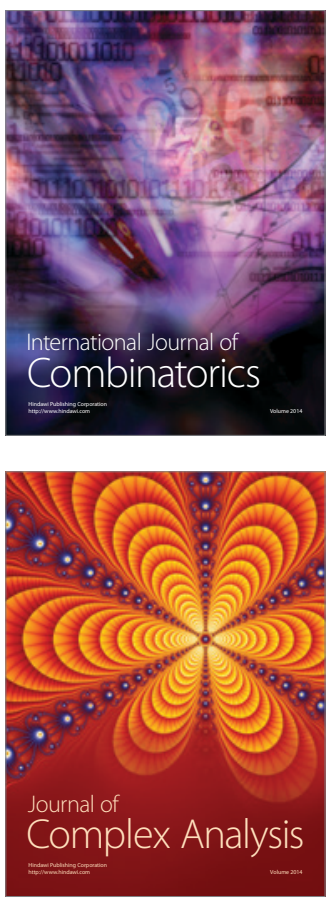

International Journal of

Mathematics and

Mathematical

Sciences
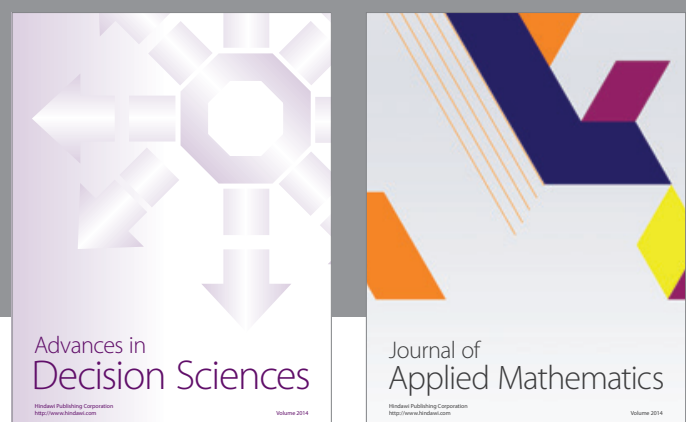

Journal of

Applied Mathematics
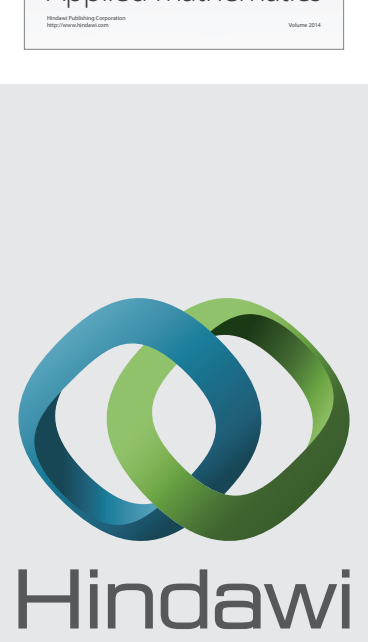

Submit your manuscripts at http://www.hindawi.com
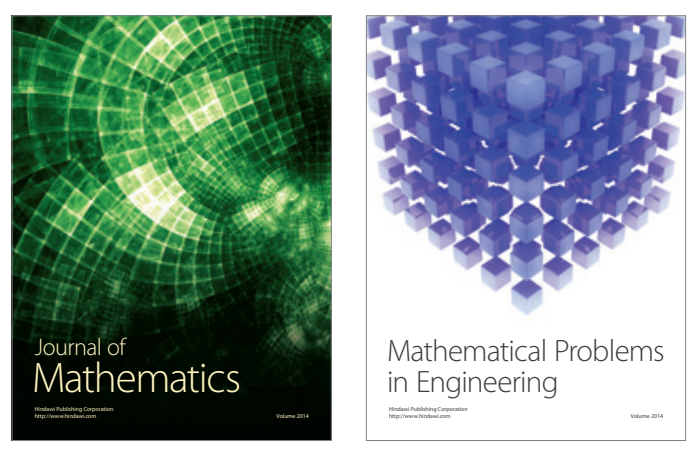

Mathematical Problems in Engineering
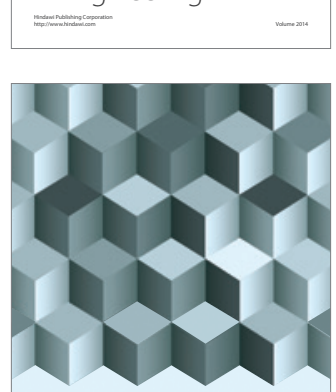

Journal of

Function Spaces
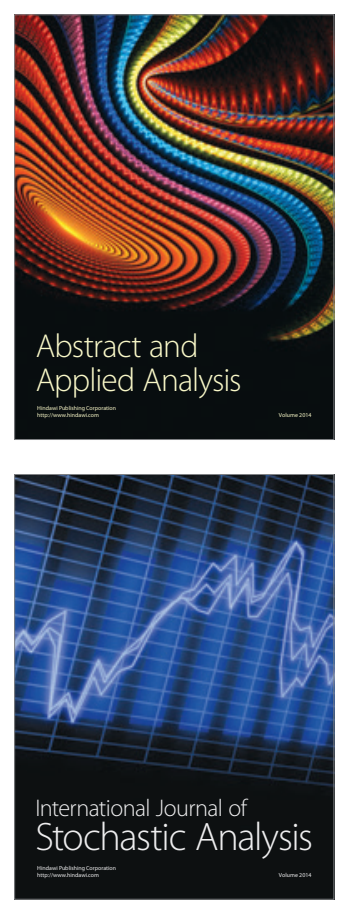

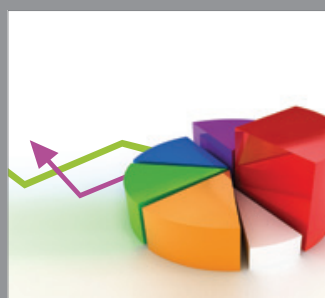

ournal of

Probability and Statistics

Promensencen
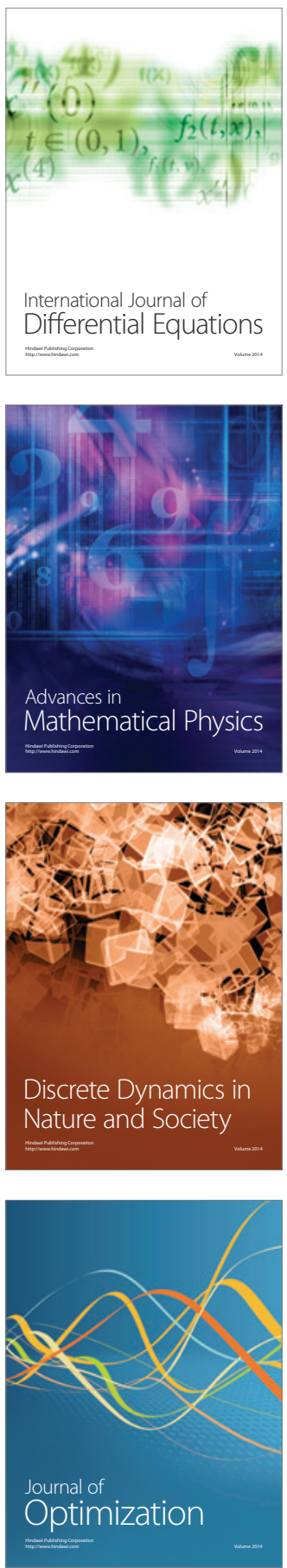